\title{
何D

3-19-2019

\section{Association of Reported Fish Intake and Supplementation Status with the Omega-3 Index}

Kristina H. Jackson

Jason M. Polreis

Nathan L. Tintle

Dordt College, nathan.tintle@dordt.edu

Penny M. Kris-Etherton

Pennsylvania State University

William S. Harris

University of South Dakota

Follow this and additional works at: https://digitalcollections.dordt.edu/faculty_work

Part of the Dietetics and Clinical Nutrition Commons

\section{Recommended Citation}

Jackson, K. H., Polreis, J. M., Tintle, N. L., Kris-Etherton, P. M., \& Harris, W. S. (2019). Association of Reported Fish Intake and Supplementation Status with the Omega-3 Index. Prostaglandins, Leukotrienes and Essential Fatty Acids, 142, 4. https://doi.org/10.1016/j.plefa.2019.01.002

This Article is brought to you for free and open access by Dordt Digital Collections. It has been accepted for inclusion in Faculty Work Comprehensive List by an authorized administrator of Dordt Digital Collections. For more information, please contact ingrid.mulder@dordt.edu. 


\title{
Association of Reported Fish Intake and Supplementation Status with the Omega-3 Index
}

\author{
Abstract \\ Background: An Omega-3 Index (O3l; EPA+DHA as a \% of erythrocyte total fatty acids) in the desirable \\ range ( $8 \%-12 \%)$ has been associated with improved heart and brain health. \\ Objective: To determine the combination of fish intake and supplement use that is associated with an 03। \\ of $>8 \%$. \\ Design: Two cross-sectional studies comparing the $03 \mathrm{I}$ to EPA+DHA/fish intake. \\ Participants/setting: The first study included 28 individuals and assessed their fish and EPA+DHA intake \\ using both a validated triple-pass 24 -hr recall dietary survey and a single fish-intake question. The second \\ study used de-identified data from 3,458 adults ( $84 \%$ from US) who self-tested their O3I and answered \\ questions about their fish intake and supplement use.
}

Statistical analyses performed: Study 1, chi-squared, one-way ANOVA, and Pearson correlations were computed. In Study 2, multi-variable regression models were used to predict 03I levels from reported fish/ supplement intakes.

Results: The mean \pm SD 03I was $4.87 \pm 1.32 \%$, and $5.99 \pm 2.29 \%$ in the first and second studies, respectively. Both studies showed that for every increase in fish intake category the 031 increased by $0.50-0.65 \%(p<0.0001)$. In the second study, about half of the population was taking omega-3 supplements, $32 \%$ reported no fish intake and $17 \%$ reported eating fish $>2$ times per week. Taking an EPA+DHA supplement increased the $03 \mathrm{I}$ by $2.2 \%(p<0.0001)$. The odds of having an $03 \mathrm{I}$ of $\geq 8 \%$ were $44 \%$ in the highest intake group ( $\geq 3$ servings/week and supplementation) and $2 \%$ in the lowest intake group (no fish intake or supplementation); and in those consuming 2 fish meals per week but not taking supplements (as per recommendations), 10\%.

Conclusion: Current AHA recommendations are unlikely to produce a desirable 03I. Consuming at least 3 fish servings per week plus taking an EPA+DHA supplement markedly increases the likelihood of achieving this target level.

\section{Keywords}

omega-3 fatty acids, omega-3 index, eicosapentaenoic acid, docosahexaenoic acid, food frequency questionnaire, dietary records, fish, dietary supplements

\section{Disciplines}

Dietetics and Clinical Nutrition

\section{Comments}

Online article information:

https://www.sciencedirect.com/science/article/abs/pii/S0952327818302254\#! 
1 TITLE: Association of Reported Fish Intake and Supplementation Status with the Omega-

23 Index

3 AUTHORS: K. H. Jacksona, J. M. Polreisa, N. L. Tintleb, P. M. Kris-Ethertonc, W. S. Harrisa,d

4 AFFILIATIONS: aOmegaQuant, LLC, Sioux Falls, SD; bDepartment of Mathematics and

5 Statistics, Dordt College, Sioux Center, IA; cDepartment of Nutritional Sciences, The

6 Pennsylvania State University, University Park, PA; dSanford School of Medicine - University

7 of South Dakota, Sioux Falls, SD

8 Corresponding Author:

9 Kristina H. Jackson, PhD, RD

10 OmegaQuant, LLC. 5009 W. 12th st, Suite 8, Sioux Falls, SD 57106

$11 \quad 605-271-6917$

12 kristina@omegaquant.com

13

14 Source of support. This research did not receive any specific grant from funding agencies in the 15 public, commercial, or not-for-profit sectors.

16 Disclosures. KHJ and JMP are employees, and WSH is the President of OmegaQuant, LLC, a

17 commercial laboratory that offers the Omega-3 Index test. NLT and PMKE have nothing to 18 disclose. 
21 Background. An Omega-3 Index (O3I; EPA+DHA as a \% of erythrocyte total fatty acids) in the

22 desirable range (8\%-12\%) has been associated with improved heart and brain health.

Objective. To determine the combination of fish intake and supplement use that is associated

24 with an $\mathrm{O} 3 \mathrm{I}$ of $>8 \%$.

Design. Two cross-sectional studies comparing the O3I to EPA+DHA/fish intake.

26 Participants/setting. The first study included 28 individuals and assessed their fish and

27 EPA+DHA intake using both a validated triple-pass 24-hr recall dietary survey and a single fish-

28 intake question. The second study used de-identified data from 3,458 adults ( $84 \%$ from US) who

29 self-tested their O3I and answered questions about their fish intake and supplement use.

30 Statistical analyses performed. Study 1, chi-squared, one-way ANOVA, and Pearson correlations

31 were computed. In Study 2, multi-variable regression models were used to predict O3I levels

32 from reported fish/supplement intakes.

33 Results. The mean \pm SD O3I was $4.87 \pm 1.32 \%$, and $5.99 \pm 2.29 \%$ in the first and second studies,

34 respectively. Both studies showed that for every increase in fish intake category the O3I

35 increased by $0.50-0.65 \%(\mathrm{P}<0.0001)$. In the second study, about half of the population was

36 taking omega-3 supplements, $32 \%$ reported no fish intake and $17 \%$ reported eating fish $>2$ times

37 per week. Taking an EPA+DHA supplement increased the O3I by $2.2 \%(\mathrm{P}<0.0001)$. The odds of

38 having an O3I of $\geq 8 \%$ were $44 \%$ in the highest intake group ( $\geq 3$ servings/week and

39 supplementation) and $2 \%$ in the lowest intake group (no fish intake or supplementation); and in

40 those consuming 2 fish meals per week but not taking supplements (as per recommendations), $41 \quad 10 \%$. 
42 Conclusions. Current AHA recommendations are unlikely to produce a desirable O3I.

43 Consuming at least 3 fish servings per week plus taking an EPA+DHA supplement markedly

44 increases the likelihood of achieving this target level.

45 Keywords: omega-3 fatty acids, omega-3 index, eicosapentaenoic acid, docosahexaenoic acid,

46 food frequency questionnaire, dietary records, fish, dietary supplements

47 Abbreviations: American Heart Association, AHA; eicosapentaenoic acid, EPA

48 docosahexaenoic acid, DHA; fatty acid, FA; Omega-3 Index, O3I (EPA+DHA as a percent of

49 total erythrocyte FAs).

50 Author contributions. All authors participated in (1) the conception and design of the study, or

51 acquisition of data, or analysis and interpretation of data, (2) drafting the article or revising it

52 critically for important intellectual content, and (3) final approval of the version to be submitted. 
In 2018, the American Heart Association (AHA) updated its 2002 recommendations

55 regarding fish and seafood consumption from “...a variety of (preferably oily) fish at least twice a week" [1] to "...1 to 2 seafood meals per week" [2]. This apparent downgrade in the recommendation (i.e., removal of "preferably oily" and "at least") was made despite evidence that consuming fish more frequently (such as daily or multiple times per day) may impart even greater cardioprotection [3-5]. An online commentary by Kuller that accompanied the publication of the new AHA guidelines questioned whether the new fish intake recommendations would produce cardioprotective blood omega-3 levels [6]. He argued that intake recommendations should be based on those that achieve a target blood level.

Asking individuals about their fish intake is often a proxy measure for intake of omega-3 fatty acids (FAs) eicosapentaenoic (EPA) and docosahexaenoic acids (DHA), because, in nature, EPA and DHA are found almost exclusively in oily fish, such as salmon, herring and mackerel. Blood levels of EPA and DHA have been shown to be related to reported fish intake [7] and supplementation [8], and to cardiovascular health [9], a single biomarker representing both dietary intake and risk for disease.

The Omega-3 Index (O3I) is a measure of the proportion of EPA and DHA in erythrocytes (EPA+DHA/total FAs) and was originally proposed as a risk factor for cardiac death in 2004 by Harris and Von Schacky [10]. Recently, a pooling study of 10 cohort studies confirmed that an $\mathrm{O} 3 \mathrm{I}$ of $8 \%$ was related to a risk reduction of $35 \%$ for cardiovascular death, as compared to $4 \%$ (typical O3I in low-fish intake individuals) [9]. While the O3I is a significant predictor of fatal cardiovascular events, research has shown that higher blood omega-3 levels are 
75 beneficially related to other aging-related health conditions also, such as congitive function [11] 76 and brain volume [12] and increased longevity [13, 14].

77 There is some controversy regarding the effects of fish intake on the O3I. For example, 78 Block et al reported that individuals reporting an intake of at least 2 fish meals per week had an 79 O3I of 5.1\% [7], and Harris et al. found that after 4 months of consuming 2 oily fish meals per 80 week, the mean O3I was 6.1\% [15]. On the other hand, Sands et al. reported a mean O3I of 8\% 81 in non-supplementing subjects reporting this weekly intake [16]. So, whether a diet including 82 only 2 servings of fish per week would result in a cardioprotective O3I (8\%) or not is unclear. 83 To address this question, we conducted two studies. The first was a small but intensive study 84 using standard dietary intake tools to quantify both fish and EPA+DHA intake and then to 85 correlate these with the O3I. The second was conducted in a "real-world" setting to determine 86 the associations between self-reported intake of fish and/or omega-3 (i.e., EPA and DHA) 87 supplements and the O3I. 


\section{SUBJECTS AND METHODS}

\subsection{Study 1}

The study utilized a cross-sectional design with one clinic visit and three, 24-hour dietary recalls collected within two weeks of the visit. A sample of generally healthy adults was recruited by email and fliers in two sites, State College, PA and Sioux Falls, SD, US, and screened via telephone or in person during the summer of 2009. Eligibility criteria included generally healthy men and women, aged 19-65 years, BMI 19-40 kg/m2, not taking any fish oil or other EPA or DHA-containing supplements within the last 2 months, not taking flaxseed oil supplements within the last week, and having a stable diet pattern, especially with respect to seafood, for the previous 6 months. Those who were ineligible were pregnant or nursing, ill, taking prescription medications, smokers, or did not fit into an available fish intake group (see below). Thirty participants were recruited; 28 were eligible and had reasonable responses on the questionnaires. The study visit consisted of a finger prick to collect a dried blood spot, a short questionnaire, and measuring height and weight. The Institutional Review Boards at both sites (Penn State and Sanford Research, Sioux Falls, SD) approved all study procedures.

\subsubsection{Dietary Assessment}

The dietary assessment consisted of triplicate 24-hour dietary recalls, and a single screening question derived from the Cardiovascular Health Study [17]. Three dietary recalls were collected via telephone by trained interviewers at The Pennsylvania State University Diet Assessment Center within 2 weeks of the clinic visit. Dietary intake data were analyzed by using the Nutrition Data System for Research software, version 2009, developed by the Nutritional Coordinating Center at the University of Minnesota, Minneapolis, Minnesota. Diet recalls were conducted on unannounced, random, non-consecutive days with at least one weekend day of data 
112 by using a multi-pass methodology $[18,19]$. At the clinic visit, a single screening question was

113 used to estimate the person's perceived, average intake of "fatty" fish [7]. The screening question

114 was, "How often do you eat — as a main course — tuna or other non-fried fish?" Possible

115 responses were a) 1 or fewer times per month, b) 2-3 times per month, c) 1 time per week, d) 2

116 times per week, or e) more than 2 times per week. A serving was defined as 3 ounces to the

117 participants. Recruitment continued until six participants in each of the five fish intake

118 categories above had been enrolled.

1192.2 Study 2

120 Data were derived from 3,458 individuals who 1) sent in a dried blood spot to

121 OmegaQuant, LLC (Sioux Falls, SD, US) for testing between March 30, 2017 - January 15,

122 2018, 2) answered questions regarding their fish and omega-3 supplement intake (see below),

123 and 3) were at least 18 years of age. Individuals purchased O3I tests directly online, or they were

124 tested at conferences or expositions, by their health care provider, or in workplace screenings.

125 Identifying information (names, physical addresses [except state and country], email addresses,

126 phone numbers) were removed from the dataset. The dataset contained samples from 28

127 countries. Approval to use de-identified, existing data was obtained from the University of South

128 Dakota Institutional Review Board.

129 2.2.1 Dietary and Demographic Information

Individuals completed a short form included in their blood sample collection kit. This

131 form included personal contact information for returning results as well as some demographic

132 (age, sex, country) and dietary intake information. The fish-related dietary question was the same

133 as asked in Study 1, however the possible responses were slightly different: "None per week,"

134 "Every other week," "Every week," "2 times per week," and "3 or more times per week." The 
135 supplement-related questions were as follows: "Do you take an omega-3 supplement?" with the

136

137

138 responses: "Yes" or "No." If yes, they were asked which kind of supplement: "Fish oil," "Krill oil," “Algal oil," and "Flaxseed oil." The supplements with EPA+DHA (krill, fish, and algal oils; $\mathrm{n}=1,681$ ) were included in the "EPA+DHA supplement" category, but individuals who reported taking flaxseed oil $(n=45)$ or did not report the kind of supplement $(n=75)$ were excluded from this analysis.

\subsection{Omega-3 Index Analysis}

In both studies, an O3I kit was used to collect a dried blood spot as previously described [20]. After receipt in the laboratory, capillary column gas chromatography was used with an internal-standard-based, three-point calibration curve to quantify levels of 24 FAs. Blood spots were transferred to a reaction vial. FA methyl esters were generated using boron trifluoride in methanol as a methylation reagent. Samples were heated for $45 \mathrm{~min}$ at 100C, extracted into hexane (after the addition of water) and analyzed using a GC2010 Gas Chromatograph (Shimadzu Corporation, Columbia, MD) equipped with a SP2560, 100-m column (Supelco, Bellefonte, PA). FA were identified by comparison with a standard mixture of FA (GLC, Nucheck Prep, Elysian, MN). The O3I (an erythrocyte-specific metric) was calculated from the dried blood spot EPA+DHA value using an equation derived by comparing values in 98 random samples and is expressed as a percent of total FAs. The correlation coefficient between O3I and the dried blood spot EPA+DHA was $0.96(\mathrm{P}<0.0001)$. The laboratory coefficient of variation for the $\mathrm{O} 3 \mathrm{I}$ is $<5 \%$. All individual FA, including EPA and DHA, are whole blood levels.

\subsection{Statistical Analyses}

In study 1, demographic characteristics were compared across fish intake groups using chi-squared and one-way ANOVA methods, with p-values also estimated using a resampling 
158 approach to ensure robustness due to small sample sizes within each category. Pearson

159 correlations were computed between the O3I and both the frequency of intake (1 question) and

160 the calculated EPA+DHA intake from the dietary recalls, with multiple regression models used

161 to estimate correlations after adjusting for demographic factors (BMI, age, sex, race). In study 2,

162 multiple regression models were used to estimate the adjusted effects of intake frequency and

163 supplementation on blood FA levels, after adjusting for demographic covariates. Seventy-five

164 percent prediction intervals are calculated in order to provide estimates of the range of O3I

165 values based on reported fish consumption, supplement and age, based on a multiple regression

166 model. A significance level of 0.05 and two-sided tests were used for all analyses which were

167 run using $\mathrm{R}$ version 3.5 (www.r-project.org). 


\section{RESULTS}

169

170

171

172

173

174

175

176

177

178

179

180

181

182

183

184

185

186

187

188

189

190

\subsection{Study $1 \quad(\mathrm{n}=28)$}

Demographic characteristics were not significantly different among the five fish intake groups (Table 1). Overall, the mean age was 32 years and BMI, $24.3 \mathrm{~kg} / \mathrm{m} 2$. All participants were from the US, $82 \%$ were Caucasian, and $75 \%$ were female (Table 1). Based on the 24-hour dietary recall data, the most commonly eaten fish was tuna (32\% of fish eaten) followed by salmon (16\%), both of which are species known for their high EPA+DHA content. Eight other varieties of fish and shellfish (cod, imitation crab, shrimp, haddock, catfish, swordfish, mussel, sardines) were significant contributors of EPA+DHA (based on providing at least $0.05 \mathrm{~g}$ EPA+DHA in the meal- not energy adjusted). Estimated overall EPA+DHA intakes were 300 mg/d (assuming $2000 \mathrm{kcal}$ intake, Table 1), ranging from about 34 to $620 \mathrm{mg} /$ day across fish intake categories.

The association between the O3I (and whole blood EPA and DHA separately) and both the five fish intake groups and the calorie-adjusted intake of EPA and DHA were significant. For the former comparison, the Pearson correlation coefficients were 0.48 for EPA $(\mathrm{p}=0.009), 0.59$ for DHA $(\mathrm{p}<0.001)$ and 0.61 for the O3I $(\mathrm{p}<0.001)$. There was an estimated 0.6 percentage point increase in the O3I for each additional fish consumption category (Table 2). In a multivariable model containing all four subject demographic factors and reported fish consumption frequency, only the latter was a significant predictor of the O3I. The associations between the O3I and calculated EPA and DHA intakes were weaker than those comparing the former with fish intake frequency. Correlations between blood FAs and calculated intakes were 0.26 for EPA $(p=0.17)$, 0.44 for DHA $(\mathrm{p}=0.02)$, and 0.42 for the O3I $(\mathrm{p}=0.03)$. Correlations between fish frequency group and calculated EPA+DHA intakes were 0.61 (EPA), 0.59 (DHA) and 0.61 (EPA+DHA). 
191

The mean age of this cohort was 51 years, over half were women (60\%), and they were primarily from the US (84\%) (Table 3). Approximately half of the sample reported taking an EPA+DHA supplement at the time of the study (includes fish, krill and algal oil supplements). Slightly less than one third of the sample reported never eating fish, with another similarly sized group reporting each fish approximately every other week, and the remaining individuals reporting fish consumption at least weekly (38\%). About $83 \%$ of individuals had an O3I below the desirable range of $8 \%-12 \%, 16 \%$ had values within that range, and $2 \%$ had values above.

Individuals taking EPA+DHA supplements and/or eating fish more frequently had higher O3I values (Figure 1, Table 4). Furthermore, older individuals and individuals from outside of the US tended to have higher O3I values (Table 4). All variables remained statistically significant in a multi-variable model predicting O3I values, with an estimated increase of $2.2 \%$ when taking an EPA+DHA supplement, and an estimated $0.6 \%$ increase for each additional fish meal per week. The multi-variable model explained $32.7 \%$ of the variance in the O3I.

Using a multivariable model including only age, reported frequency of fish intake, and omega-3 supplement use, a hypothetical 50-yr old person who did not take supplements and reported no fish intake would be predicted to have an O3I of about $4.5 \%$ (75\% prediction interval [PI]: $2.3 \%$ to $6.7 \%$.) At the other extreme, the same individual reporting both supplement use and three or more fish meals per week would have an O3I of 8.6\% (75\% PI, $6.4 \%$ to $10.8 \%$ ) (Table 5 ). 


\section{DISCUSSION}

The overall purpose of these studies was to answer the question, "How much EPA+DHA

213 from fish and/or supplements is needed to achieve a desirable O3I?" Using the answers to two

214 simple dietary questions - "How often to you eat tuna or other non-fried fish?" and, "Do you

215 take an omega-3 supplement?" - allowed us (along with age) to begin to answer that question.

Two studies were reported here. The aim of the first study was to determine whether the

217 answer to a simple fish intake question was as good as a calculated EPA+DHA intake from an

218 intensive, triple 24-hr recall intake survey, with regards to their correlations with the O3I. We

219 found stronger correlations with the O3I for the one question than for the more rigorous (but apparently less useful) recall method. The reason for this is likely that for nutrients (like EPA

221 and DHA) that are provided in high amounts by a very small number of foods, a 3-day diet

222 record is very unlikely to capture the "true" average intake. If the fish meal day happens to be

223 included in the 3 days, the overall average intake will be over-estimated; if the fish meal is not

224 eaten in that 3 day window, the average intake will be under-estimated. This increases variability

225 and thus lowers correlations with biomarkers that actually do represent an average intake. The

226 aim of the second study was to determine, in a large cohort of people who self-reported fish and

227 supplement intake, what combination of these two sources of EPA and DHA was required

228 achieve an O3I of $>8 \%$. We found that those with the best chance (44\%) of achieving an

229 desirable O3I were reporting the consumption of at least 3 fish meals a week and were taking an

230 EPA+DHA supplement.

It is of interest to know how much EPA+DHA would be provided by $>2$ fish meals/wk +

232 supplementation. We estimated this using the following assumptions: 1) a serving of oily fish

233 provides about 1,250 mg EPA+DHA (average amount provided by 4 oz of wild coho salmon, 
234 Bluefin tuna, sardines and Albacore tuns, from USDA Nutrient Database[21]), and 2) a standard 235 fish oil capsule contains about $300 \mathrm{mg}$ EPA+DHA. Based on these values, amount of

236 EPA+DHA from fish for 3 servings per week would be 3,750 mg/wk (or $535 \mathrm{mg}$ EPA+DHA per

237 day). Add $300 \mathrm{mg}$ EPA+DHA per day from one supplement, and the overall intake equals

238 around $835 \mathrm{mg}$ EPA+DHA per day. This is approximately how much EPA+DHA one would

239 need to consume in the long-term to achieve an average O3I of $8 \%$, but it is $>3$ times the

240

EPA+DHA recommended by the Dietary Guidelines for Amercians (250 mg/day) [22], and 1.7

times that recommended by the Academy of Nutrition and Dietitics (500 mg/day) [23]. It is also

242 about 8 times the typical EPA+DHA intake in the US ( 100 mg/day) [24]. Only about $10 \%$ of Americans eat as much as 2 fish meals per week [DGAC 2015]. Not surprisingly, an estimated 95\% of Americans do not have optimal O3I levels [25]. In 2007 we reported that 2 fish meals per week (salmon and albacore tuna) provided an average of $485 \mathrm{mg}$ EPA+DHA per day, and after months of this regimen, the mean O3I increased from $4 \%$ to $6.2 \%$ [15]. This observation further underscores the inability of the AHA recommendations to produce an optimal O3I.

Therefore, there is a discrepancy between the amount of EPA+DHA provided by current fish intake recommendations $(250-500 \mathrm{mg} / \mathrm{d})$ vs. the amount needed by most Americans to reach an $\mathrm{O} 3 \mathrm{I}$ of $8 \%$ (>800 mg/d, according to our calculations). As Kuller wrote in a commentary on the 2018 AHA fish intake guidelines, "The key public health question is whether the

252 recommended intake of seafood in the US should be [set so as] to reach the same levels of n-3 253 PUFAs in blood as in Japan, about 9\% of total FAs in blood versus 4\% in the US [26]." Based on 254 this rationale (and our data), at least 2-3 servings per week of oily fish, rather than "1-2 servings 255 of seafood" [2], should be the recommendation. But even this will not produce an O3I of $>8 \%$ to achieve that, either adding an EPA+DHA supplement or increasing to 4-5 servings of oily fish 257 per week would be necessary. 
What was the basis for the AHA's recommendation of "1-2 seafood meals per week?"

Rimm et al.[2] stated that "...there is little additional benefit in risk reduction with a higher intake [than 1-2 servings per week]." This, in our view, does not reflect the current state of the data. Considerable evidence supports the view that higher fish intakes and higher blood levels of omega-3s are associated with significant additional reduction in cardiovascular risk $[3,5,27$, 28], in a dose-dependent manner with no plateau at 1-2 servings per week. The biggest reduction in risk may well occur between zero servings and 1-2 servings per week, but to suggest that there is no additional benefit at high intakes and blood levels ignores a substantial body of evidence cited above. We do recognize that public health recommendations must balance what is ideal vs. what is practical for the public, and also must take into consideration other non-nutritional factors, i.e. potentially hazadous components of fish (mercury, PCBs) and the sustainability of the world's fish supply. However, the basis for concluding that there is "no evidence" that higher fish intakes are associated with improved outcomes is obscure at best.

Why is an $\mathrm{O} 3 \mathrm{I}$ of $8 \%$ the desirable level? The O3I was originally presented as a risk factor for cardiac death, which is where the cut-offs for desirable $(>8 \%)$ and undesirable $(<4 \%)$ [10] were estimated from the data available prior to 2004. Recently, a meta-analysis from 10 prospective cohort studies has confirmed that an $8 \%$ O3I was associated with a $35 \%$ reduction in risk for fatal CHD compared to an Index of $<4 \%[9,27]$. In another report, a level of $8 \%$ or greater (extrapolated from whole blood EPA+DHA levels) was significantly associated with the slowest rate of telomere attrition [13], and in the Women's Health Initiative Memory Study, those with an Index of $8 \%$ or greater had a $31 \%$ lower risk of death from any cause over the ensuing 15 years [13]. Similarly, individuals with a O3I of $7.8 \%$ (median of the highest quintile in the Framingham Offspring Study) were 34\% less likely to die from any cause over 7 years of follow-up compared to those with an Index of 3.7\% (median of the lowest quintile) [28]. In 
addition, an Index at this level was linked with better cognitive performance [11], improved depressive symptoms [29, 30], better cardiac remodeling post myocardial-infarction [31], lower odds of being an acute coronary syndrome patient [32], improved arthritis symptoms [33], and better cognitive function/brain size in B-vitamin-treated subjects [34]. This is also the range typical of the traditional Japanese [35], a population with a very low CHD rate and one of the longest life-expectancies in the world [36]. Finally, as emphasized by Myer and DeGroot, RCTs which achieved an $\mathrm{O} 3 \mathrm{I}$ of $8 \%$ were more likely to see CVD benefits [37]. Thus, there is substantial evidence supporting a target of $8 \%$ or more as optimal.

Although an $\mathrm{O} 3 \mathrm{I}$ of $8 \%$ is a reasonable therapeutic target, is it realistic? Can it be achieved with diet alone? Clearly, it can, again based on the Japanese experience. There, the median fish consumption is $\sim 3$ fish servings per week, resulting in an estimated $750-1,000 \mathrm{mg}$ EPA+DHA per day $[4,38]$. Interestingly, this intake is similar to the $\sim 835 \mathrm{mg} /$ day calculated above for 3 fish meals + supplementation. The average erythrocyte EPA+DHA levels in Japan range from $6.8-9.0 \%$, depending on the study and population $[4,38,39]$. So, yes, an O3I $>8 \%$ is achievable by diet alone. But Japan is fairly unique. In the US the average fish intake is less than 1 serving per week which provides approximately $100 \mathrm{mg}$ EPA+DHA per day [24]). The average O3I for Americans ranges from 4\%-6\% [25]. So, short of adopting the Japanese diet (for a lifetime), it appears that taking an EPA+DHA supplement could be an important for achieving a cardioprotective O3I. The O3I calculator (https://omegaquant.com/omega-3-calculator/) is a useful tool to roughly estimate how much more EPA+DHA one needs to eat in order to achieve a desirable O3I level based on their current levels and intake [8]. For example, according to the calculator, a man with an O3I of $4.5 \%$ would need an estimated $951 \mathrm{mg}$ EPA+DHA per day to reach $8 \%$. To get $~ 950 \mathrm{mg}$ EPA+DHA per day, he could start by eating three wild sockeye salmon meals per week (at 974 mg EPA+DHA per 4-oz. serving), which would amount to 417 
mg EPA+DHA per day [21]. Then he could add at least $500 \mathrm{mg}$ EPA+DHA per day through taking a supplement, for a total of $917 \mathrm{mg}$ EPA+DHA per day. Alternatively, he could achieve this intake by eating oily fish almost every day: two servings of sockeye salmon (974 mg EPA+DHA per $4 \mathrm{oz}$ serving), two cans of Albacore tuna (1483 mg EPA+DHA) and two cans of sardines (903 mg EPA+DHA) per week. Or finally, he could eat no fish at all and take 3 standard fish oil capsules per day (900 mg EPA+DHA). Whatever the approach, it would need to be consistent for at least 4 months in order to reach a new steady state O3I[40].

This study had significant limitations which may have contributed to the wide variability in the $\mathrm{O} 3 \mathrm{I}$ across intake/supplementation categories. First, the single fish question was vague with regards to the actual types of fish consumed, and the supplement question did not take doses and frequency of supplementation into account. This is the price one pays for creating simple, consumer-directed questions instead of complex, research-based questions. Second, individual variability in O3I levels could be due to genetic and biological differences (age [25], genetic variants [41]) affecting the incorporation of FAs into tissue. Finally, there is always the chance that the individuals misreported their dietary intake, making the relationship between the reported intake and O3I seem incongruent on an individual basis. Therefore, these two questions, although useful at a population level, are probably less so for the individual. It should be emphasized that this cohort is in no way "representative" of the omega-3 status of each individual country from which these samples were collected. These individuals were typically attending trade shows and thus were much more likely to be interested in omega-3 FAs than the average person, and hence probably consumed more fish/fish oil. Thus, these data are not generalizable to the populations tested, but they were appropriate to address our study question. This was also a cross sectional study in which only one blood sample and only one response to the questions asked were available. Multiple data points per person would likely have reduced 
330 confirm the omega-3 status.

\section{CONCLUSION}

The current study again validates the O3I as a useful biomarker of EPA+DHA intake.

Reports of higher fish intake corresponded with higher O3I values in a dose-dependent manner. Reported supplementation with EPA+DHA (fish, krill, or algal oils) was associated with an approximately 2 percentage point higher O3I. The current fish intake recommendations (1-2 servings of seafood per week) are unlikely to produce a cardioprotective O3I level, but consuming primarily oily fish 3 times or more per week and supplementation may. Despite these strong relationships, individual variability is great, and testing blood levels is the only way to 


\section{REFERENCES}

[1] P.M. Kris-Etherton, W.S. Harris, L.J. Appel, C. American Heart Association. Nutrition. Fish consumption, fish oil, omega-3 fatty acids, and cardiovascular disease. Circulation. 106 (2002) 2747-2757.

[2] E.B. Rimm, L.J. Appel, S.E. Chiuve, L. Djousse, M.B. Engler, P.M. Kris-Etherton, D. Mozaffarian, D.S. Siscovick, A.H. Lichtenstein, L. American Heart Association Nutrition Committee of the Council on, H. Cardiometabolic, E. Council on, Prevention, Y. Council on Cardiovascular Disease in the, C. Council on, N. Stroke, C. Council on Clinical. Seafood LongChain n-3 Polyunsaturated Fatty Acids and Cardiovascular Disease: A Science Advisory From the American Heart Association. Circulation. 138 (2018) e35-e47.

[3] K. He, Y. Song, M.L. Daviglus, K. Liu, L. Van Horn, A.R. Dyer, P. Greenland. Accumulated evidence on fish consumption and coronary heart disease mortality: a meta-analysis of cohort studies. Circulation. 109 (2004) 2705-2711.

[4] H. Iso, M. Kobayashi, J. Ishihara, S. Sasaki, K. Okada, Y. Kita, Y. Kokubo, S. Tsugane. Intake of fish and $\mathrm{n} 3$ fatty acids and risk of coronary heart disease among Japanese: the Japan Public Health Center-Based (JPHC) Study Cohort I. Circulation. 113 (2006) 195-202.

[5] K. Hamazaki, H. Iso, E.S. Eshak, S. Ikehara, A. Ikeda, M. Iwasaki, T. Hamazaki, S. Tsugane, J.S. Group. Plasma levels of n-3 fatty acids and risk of coronary heart disease among Japanese: The Japan Public Health Center-based (JPHC) study. Atherosclerosis. 272 (2018) 226-232. [6] L.H. Kuller. Omega-3 fatty acids and coronary heart disease: A very fishy story. 2018. https://professional.heart.org/professional/ScienceNews/UCM_501197_Omega-3-Fatty-Acidsand-Coronary-Heart-Disease-A-Very-Fishy-Story.jsp. 7/26/2018:

[7] R.C. Block, W.S. Harris, J.V. Pottala. Determinants of Blood Cell Omega-3 Fatty Acid Content. Open Biomarkers J. 1 (2008) 1-6.

[8] M.R. Flock, A.C. Skulas-Ray, W.S. Harris, T.D. Etherton, J.A. Fleming, P.M. Kris-Etherton. Determinants of Erythrocyte Omega-3 Fatty Acid Content in Response to Fish Oil Supplementation: A Dose-Response Randomized Controlled Trial. Journal of the American Heart Association. 2 (2013) e000513.

[9] W.S. Harris, L. Del Gobbo, N.L. Tintle. The Omega-3 Index and relative risk for coronary heart disease mortality: Estimation from 10 cohort studies. Atherosclerosis. 262 (2017) 51-54. [10] W.S. Harris, C. von Schacky. The Omega-3 Index: a new risk factor for death from coronary heart disease? Prev.Med. 39 (2004) 212-220.

[11] K. Lukaschek, C. von Schacky, J. Kruse, K.H. Ladwig. Cognitive Impairment Is Associated with a Low Omega-3 Index in the Elderly: Results from the KORA-Age Study. Dementia and geriatric cognitive disorders. 42 (2016) 236-245.

[12] J.V. Pottala, K. Yaffe, J.G. Robinson, M.A. Espeland, R. Wallace, W.S. Harris. Higher RBC EPA + DHA corresponds with larger total brain and hippocampal volumes: WHIMS-MRI Study. Neurology. 82 (2014) 435-442. [13] W.S. Harris, J. Luo, J.V. Pottala, M.A. Espeland, K.L. Margolis, J.E. Manson, L. Wang, T.M. Brasky, J.G. Robinson. Red blood cell polyunsaturated fatty acids and mortality in the Women's Health Initiative Memory Study. J Clin Lipidol. 11 (2017) 250-259. [14] R. Farzaneh-Far, J. Lin, E.S. Epel, W.S. Harris, E.H. Blackburn, M.A. Whooley. Association of marine omega-3 fatty acid levels with telomeric aging in patients with coronary heart disease. JAMA. 303 (2010) 250-257. 
[15] W.S. Harris, J.V. Pottala, S.A. Sands, P.G. Jones. Comparison of the effects of fish and fishoil capsules on the $\mathrm{n} 3$ fatty acid content of blood cells and plasma phospholipids. Am J Clin Nutr. 86 (2007) 1621-1625.

[16] S.A. Sands, K.J. Reid, S.L. Windsor, W.S. Harris. The impact of age, body mass index, and fish intake on the EPA and DHA content of human erythrocytes. Lipids. 40 (2005) 343-347. [17] D. Mozaffarian, R.N. Lemaitre, L.H. Kuller, G.L. Burke, R.P. Tracy, D.S. Siscovick, S. Cardiovascular Health. Cardiac benefits of fish consumption may depend on the type of fish meal consumed: the Cardiovascular Health Study. Circulation. 107 (2003) 1372-1377. [18] J.M. Conway, L.A. Ingwersen, A.J. Moshfegh. Accuracy of dietary recall using the USDA five-step multiple-pass method in men: an observational validation study. J Am Diet Assoc. 104 (2004) 595-603.

[19] J.M. Conway, L.A. Ingwersen, B.T. Vinyard, A.J. Moshfegh. Effectiveness of the US Department of Agriculture 5-step multiple-pass method in assessing food intake in obese and nonobese women. Am J Clin Nutr. 77 (2003) 1171-1178.

[20] W.S. Harris, J. Polreis. Measurement of the Omega-3 Index in Dried Blood Spots. Annals of Clinical and Laboratory Research. 4 (2016) e1-e7.

[21] US Department of Agriculture, Agricultural Research Service, N.D. Laboratory, USDA National Nutrient Database for Standard Reference, Legacy, in, Internet, 2018 April. [22] U.S.D.o.A.a.U.S.D.o.H.a.H. Services. Dietary Guidelines for Americans, 2010. 2010. http://www.cnpp.usda.gov/Publications/DietaryGuidelines/2010/PolicyDoc/PolicyDoc.pdf. [23] P.M. Kris-Etherton, S. Innis. Position of the American Dietetic Association and Dietitians of Canada: Dietary Fatty Acids. Journal of the American Dietetic Association. 107 (2007) 15991611.

[24] Y. Papanikolaou, J. Brooks, C. Reider, V.L. Fulgoni, 3rd. U.S. adults are not meeting recommended levels for fish and omega-3 fatty acid intake: results of an analysis using observational data from NHANES 2003-2008. Nutrition journal. 13 (2014) 31. [25] W.S. Harris, J.V. Pottala, S.A. Varvel, J.J. Borowski, J.N. Ward, J.P. McConnell. Erythrocyte omega-3 fatty acids increase and linoleic acid decreases with age: observations from 160,000 patients. Prostaglandins, leukotrienes, and essential fatty acids. 88 (2013) 257-263. [26] L.H. Kuller. Omega-3 Fatty Acids and Coronary Heart Disease: A Very Fishy Story. Circulation. (2018).

[27] L.C. Del Gobbo, F. Imamura, S. Aslibekyan, M. Marklund, J.K. Virtanen, M. Wennberg, M.Y. Yakoob, S.E. Chiuve, L. Dela Cruz, A.C. Frazier-Wood, A.M. Fretts, E. Guallar, C. Matsumoto, K. Prem, T. Tanaka, J.H. Wu, X. Zhou, C. Helmer, E. Ingelsson, J.M. Yuan, P. Barberger-Gateau, H. Campos, P.H. Chaves, L. Djousse, G.G. Giles, J. Gomez-Aracena, A.M. Hodge, F.B. Hu, J.H. Jansson, I. Johansson, K.T. Khaw, W.P. Koh, R.N. Lemaitre, L. Lind, R.N. Luben, E.B. Rimm, U. Riserus, C. Samieri, P.W. Franks, D.S. Siscovick, M. Stampfer, L.M. Steffen, B.T. Steffen, M.Y. Tsai, R.M. van Dam, S. Voutilainen, W.C. Willett, M. Woodward, D. Mozaffarian, H. Cohorts for, A. Aging Research in Genomic Epidemiology Fatty, C. Outcomes Research. Omega-3 Polyunsaturated Fatty Acid Biomarkers and Coronary Heart Disease: Pooling Project of 19 Cohort Studies. JAMA Intern Med. 176 (2016) 1155-1166. [28] W.S. Harris, N.L. Tintle, M.R. Etherton, R.S. Vasan. Erythrocyte long-chain omega-3 fatty acid levels are inversely associated with mortality and with incident cardiovascular disease: The Framingham Heart Study. J Clin Lipidol. (2018).

[29] R.M. Carney, B.C. Steinmeyer, K.E. Freedland, E.H. Rubin, M.W. Rich, W.S. Harris. Baseline blood levels of omega-3 and depression remission: a secondary analysis of data from a 
placebo-controlled trial of omega-3 supplements. The Journal of clinical psychiatry. 77 (2016) e138-143.

[30] C. Horikawa, R. Otsuka, Y. Kato, Y. Nishita, C. Tange, S. Kakutani, T. Rogi, H. Kawashima, H. Shibata, F. Ando, H. Shimokata. Cross-sectional association between serum concentrations of n-3 long-chain PUFA and depressive symptoms: results in Japanese community dwellers. The British journal of nutrition. 115 (2016) 672-680. [31] B. Heydari, S. Abdullah, J.V. Pottala, R. Shah, S. Abbasi, D. Mandry, S.A. Francis, H. Lumish, B.B. Ghoshhajra, U. Hoffmann, E. Appelbaum, J.H. Feng, R. Blankstein, M. Steigner, J.P. McConnell, W. Harris, E.M. Antman, M. Jerosch-Herold, R.Y. Kwong. Effect of Omega-3 Acid Ethyl Esters on Left Ventricular Remodeling After Acute Myocardial Infarction: The OMEGA-REMODEL Randomized Clinical Trial. Circulation. 134 (2016) 378-391. [32] R.C. Block, W.S. Harris, K.J. Reid, S.A. Sands, J.A. Spertus. EPA and DHA in blood cell membranes from acute coronary syndrome patients and controls. Atherosclerosis. 197 (2008) 821-828.

[33] C. Dawczynski, M. Dittrich, T. Neumann, K. Goetze, A. Welzel, P. Oelzner, S. Volker, A.M. Schaible, F. Troisi, L. Thomas, S. Pace, A. Koeberle, O. Werz, P. Schlattmann, S. Lorkowski, G. Jahreis. Docosahexaenoic acid in the treatment of rheumatoid arthritis: A doubleblind, placebo-controlled, randomized cross-over study with microalgae vs. sunflower oil. Clin Nutr. 37 (2018) 494-504. [34] A. Oulhaj, F. Jerneren, H. Refsum, A.D. Smith, C.A. de Jager. Omega-3 Fatty Acid Status Enhances the Prevention of Cognitive Decline by B Vitamins in Mild Cognitive Impairment. Journal of Alzheimer's disease : JAD. 50 (2016) 547-557.

[35] M. Itomura, S. Fujioka, K. Hamazaki, K. Kobayashi, T. Nagasawa, S. Sawazaki, Y.

Kirihara, T. Hamazaki. Factors influencing EPA+DHA levels in red blood cells in Japan. In Vivo. 22 (2008) 131-135.

[36] The World Fact Book, Central Intelligence Agency, Washington, D.C., 2018.

[37] B.J. Meyer, R.H.M. Groot. Effects of Omega-3 Long Chain Polyunsaturated Fatty Acid Supplementation on Cardiovascular Mortality: The Importance of the Dose of DHA. Nutrients. 9 (2017).

[38] B.F. Zhou, J. Stamler, B. Dennis, A. Moag-Stahlberg, N. Okuda, C. Robertson, L. Zhao, Q. Chan, P. Elliott, I.R. Group. Nutrient intakes of middle-aged men and women in China, Japan, United Kingdom, and United States in the late 1990s: the INTERMAP study. J Hum Hypertens. 17 (2003) 623-630.

[39] T. Kawabata, S. Hirota, T. Hirayama, N. Adachi, C. Hagiwara, N. Iwama, K. Kamachi, E. Araki, H. Kawashima, Y. Kiso. Age-related changes of dietary intake and blood eicosapentaenoic acid, docosahexaenoic acid, and arachidonic acid levels in Japanese men and women. Prostaglandins Leukot Essent Fatty Acids. 84 (2011) 131-137.

[40] M.B. Katan, J.P. Deslypere, A.P. van Birgelen, M. Penders, M. Zegwaard. Kinetics of the incorporation of dietary fatty acids into serum cholesteryl esters, erythrocyte membranes, and adipose tissue: an 18-month controlled study. Journal of lipid research. 38 (1997) 2012-2022. [41] A. Kalsbeek, J. Veenstra, J. Westra, C. Disselkoen, K. Koch, K.A. McKenzie, J. O'Bott, J. Vander Woude, K. Fischer, G.C. Shearer, W.S. Harris, N.L. Tintle. A genome-wide association study of red-blood cell fatty acids and ratios incorporating dietary covariates: Framingham Heart Study Offspring Cohort. PloS one. 13 (2018) e0194882. 
479

480 
481 Table 1. Study 1 demographic, (mean [SD], n[\%])

\begin{tabular}{|c|c|c|c|c|c|c|}
\hline Characteristic & $\begin{array}{c}\text { Overall } \\
(\mathbf{n = 2 8})\end{array}$ & $\begin{array}{c}<\mathbf{1} \text { time per } \\
\text { month (n=6) }\end{array}$ & $\begin{array}{c}\text { 2-3 times per } \\
\text { month (n=6) }\end{array}$ & $\begin{array}{c}\text { 1 time per } \\
\text { week (n=5)1 }\end{array}$ & $\begin{array}{c}\text { 2 times per } \\
\text { week (n=5) }\end{array}$ & $\begin{array}{c}\text { More than 2 times } \\
\text { per week (n=6) }\end{array}$ \\
\hline \multicolumn{7}{|c|}{ Demographics } \\
\hline Sex - Male & $25 \%(7)$ & $16.7 \%(1)$ & $16.7 \%(1)$ & $20 \%(1)$ & $0 \%(0)$ & $66.7 \%(4)$ \\
\hline Age (years) & $31.6(9.5)$ & $30.3(12.0)$ & $34.2(10.3)$ & $30.2(6.8)$ & $27.0(7.5)$ & $35.3(10.2)$ \\
\hline Race - Caucasian & $82.1 \%(23)$ & $83.3 \%(5)$ & $83.3 \%(5)$ & $60 \%(3)$ & $100 \%(5)$ & $83.3 \%(5)$ \\
\hline BMI (kg/m2) & $24.3(4.5)$ & $21.9(3.4)$ & $25.9(4.8)$ & $27.0(4.9)$ & $24.2(6.0)$ & $22.9(2.7)$ \\
\hline \multicolumn{7}{|c|}{ Whole blood fatty acid levels $(\%$ composition) } \\
\hline EPA & $0.57(0.43)$ & $0.34(0.06)$ & $0.43(0.17)$ & $0.55(0.28)$ & $0.60(0.27)$ & $0.93(0.78)$ \\
\hline DHA & $3.40(0.95)$ & $2.57(0.79)$ & $2.79(0.52)$ & $3.63(0.55)$ & $4.10(0.21)$ & $4.05(1.16)$ \\
\hline Omega-3 Index1 & $4.87(1.34)$ & $3.77(0.83)$ & $4.08(0.52)$ & $5.10(0.87)$ & $5.65(0.45)$ & $5.94(1.94)$ \\
\hline \multicolumn{7}{|c|}{ Calorie-Adjusted Dietary Intake $($ g per 1000 kcal) } \\
\hline EPA & $0.05(0.07)$ & $0.004(0.002)$ & $0.02(0.03)$ & $0.06(0.04)$ & $0.05(0.04)$ & $0.12(0.12)$ \\
\hline DHA & $0.10(0.12)$ & $0.014(0.008)$ & $0.06(0.11)$ & $0.09(0.09)$ & $0.15(0.13)$ & $0.19(0.16)$ \\
\hline EPA+DHA & $0.15(0.18)$ & $0.017(0.01)$ & $0.08(0.14)$ & $0.15(0.13)$ & $0.21(0.16)$ & $0.31(0.26)$ \\
\hline
\end{tabular}

482

483 1The O3I is EPA+DHA in erythrocytes which is calculated from the EPA+DHA content of a

484 dried whole blood sample. Hence, the O3I may not equal the EPA+DHA in whole blood. 
Table 2. Association of fish intake and other characteristics with O3I levels in Study 1.

\begin{tabular}{|l|l|l|}
\hline Characteristic & \multicolumn{2}{|c|}{$\begin{array}{c}\text { Absolute percentage point difference in the O3I } \\
\text { per unit of change of the characteristic } 1\end{array}$} \\
\hline & $\begin{array}{l}\text { Bivariate } \\
\text { Beta }(95 \% \mathrm{CI})\end{array}$ & $\begin{array}{l}\text { Multivariable } 2 \\
\text { Beta }(95 \% \mathrm{CI})\end{array}$ \\
\hline $\begin{array}{l}\text { Fish consumption category } \\
\text { (meals/week) }\end{array}$ & $0.65 \%(0.31,1.00) \dagger$ & $0.56 \%(0.06,1.07) *$ \\
\hline $\begin{array}{l}\text { EPA+DHA consumption (per } \\
1000 \mathrm{kcal} \text {; log-transformed) } 2\end{array}$ & $0.33 \%(0.04,0.63)^{*}$ & $0.17 \%(-0.24,0.57)$ \\
\hline Sex- Male & $0.49 \%(-0.72,1.71)$ & $-0.32 \%(-1.50,0.87)$ \\
\hline Age (per decade) & $-0.22 \%(-0.75,0.33)$ & $-0.20 \%(-0.72,0.29)$ \\
\hline Caucasian Race & $0.05 \%(-1.33,1.45)$ & $-0.18 \%(-1.42,1.04)$ \\
\hline BMI $(\mathrm{kg} / \mathrm{m} 2)$ & $-0.07 \%(-0.18,0.05)$ & $-0.05 \%(-0.18,0.07)$ \\
\hline
\end{tabular}

$\dagger \mathrm{P}<0.0001 ; * \mathrm{P}<0.01$

$488 \quad 1$ Values in this table are beta coefficients and are interpreted as the change in O3I for a one-unit 489 change in the characteristic, e.g. for each additional fish meal per week the estimated effect on 490 O3I is 0.65 percentage point increase before and 0.56 percentage point increase after adjusting 491 for other characteristics. 2 Model $\mathrm{R}_{2}$ is 0.45 . Equation: $\mathrm{O} 3 \mathrm{I}=6.62 \%+0.56^{*}$ Estimated fish meals 492 per week $+0.17 * \log ($ EPA+DHA $)-0.32 *$ Male-0.20*(Age/10)-0.18*White-0.05*BMI. 
Table 3. Demographics, fish intake, supplementation status and blood fatty acids from

496 study $2(\mathbf{n}=\mathbf{3 4 5 8})$

\begin{tabular}{|l|l|}
\hline Characteristic & n (\%) or Mean (SD) \\
\hline Demographics & \\
\hline Age & $51.3(17.0)$ \\
\hline Sex - Male & $40.2 \%(1391)$ \\
\hline Country - USA & $84.2 \%(2912) 1$ \\
\hline Supplementation & \\
\hline None & $47.9 \%(1657)$ \\
\hline Any & $52.1 \%(1800)$ \\
\hline Fish (EPA/DHA) oil & $43.6 \%(1508)$ \\
\hline Flaxseed (ALA) oil & $1.3 \%(45)$ \\
\hline Krill (EPA/DHA) oil & $3.2 \%(111)$ \\
\hline Algal (EPA/DHA) oil & $1.8 \%(62)$ \\
\hline Unknown & $2.1 \%(74)$ \\
\hline Reported fish intake & \\
\hline None & $31.5 \%(1090)$ \\
\hline Every other week & $30.9 \%(1067)$ \\
\hline Weekly & $20.6 \%(714)$ \\
\hline Twice per week & $10.2 \%(353)$ \\
\hline Three or more times per week & $6.8 \%(234)$ \\
\hline O3I value & \\
\hline Less than 8\% & $82.6 \%(2855)$ \\
\hline $8-12 \%$ & $15.6 \%(540)$ \\
\hline Above 12\% & $1.8 \%(63)$ \\
\hline Non-US indiduas (n=546), were from & \\
\hline
\end{tabular}

1. Non-US individuals $(\mathrm{n}=546)$, were from 27 other countries (Singapore (110), India (87), Chile (58), New Zealand (36), Canada (33), Australia (33), Korea (24), Japan (21), United Kingdom (19), Norway (19), Philipines (17), Hong Kong (14), South Africa (13), China (8), Taiwan (5), Sweden (4), Denmark (4), United Arab Emirates (3), Switzerland (2), Pakistan (2), Iceland (2), Uruguay (1), Saudia Arabia (1), Netherlands (1), Mexico (1), Brazil (1) and Brunei (1).

Twenty-six individuals had an unknown country of origin. 
Table 4. Association between demographic, dietary and supplementation status and O3I values in study 2

\begin{tabular}{|c|c|c|}
\hline \multirow[t]{2}{*}{ Characteristic } & \multicolumn{2}{|c|}{$\begin{array}{c}\text { Absolute percentage point increase in O3I per unit of } \\
\text { change } 1\end{array}$} \\
\hline & $\begin{array}{l}\text { Bivariate } \\
\text { Beta }(95 \% \mathrm{CI})\end{array}$ & $\begin{array}{l}\text { Multivariable } 2 \\
\text { Beta }(95 \% \mathrm{CI})\end{array}$ \\
\hline \multicolumn{3}{|l|}{ Reported fish meals/week } \\
\hline Never & -- & -- \\
\hline Bi-weekly & $0.49 \%(0.31 \%, 0.68 \%) \dagger$ & $0.29 \%+(0.12 \%, 0.45 \%)$ \\
\hline Weekly & $1.08 \%(0.87 \%, 1.28 \%) \dagger$ & $0.65 \% \dagger(0.46 \%, 0.83 \%)$ \\
\hline Twice weekly & $1.68 \%(1.42 \%, 1.94 \%) \dagger$ & $1.18 \% \dagger(0.95 \%, 1.41 \%)$ \\
\hline Three or more per week & $2.57 \%(2.27 \%, 2.88 \%) \dagger$ & $1.90 \% *(1.63 \%, 2.18 \%)$ \\
\hline EPA/DHA Supplement - Yes & $2.36 \%(2.23 \%, 2.50 \%) \dagger$ & $2.16 \%+(2.03 \%, 2.29 \%)$ \\
\hline Sex - Male & $-0.09 \%(-0.25 \%, 0.06 \%)$ & $-0.10 \%(-0.23 \%, 0.04 \%)$ \\
\hline Age (years/10) & $0.22 \% \dagger(0.18 \%, 0.27 \%)$ & $0.09 \% \dagger(0.05 \%, 0.13 \%)$ \\
\hline Country - Not USA3 & $0.30 \% *(0.09 \%, 0.51 \%)$ & $0.45 \% \dagger(0.27 \%, 0.63 \%)$ \\
\hline
\end{tabular}

$\dagger \mathrm{P}<0.0001, * \mathrm{P}<0.01$

1 Values in this table are beta coefficients and are interpreted as the percentage point change in O3I for a one-unit change in the characteristic, e.g. for each additional fish meal per week the estimated effect on $\mathrm{O} 3 \mathrm{I}$ is $0.6 \%$ (or a 0.6 percentage point increase) both before and after adjusting for other characteristics.

${ }_{2}$ Overall model $\mathrm{R}_{2}$ is 0.33 .

Equation: $\mathrm{O} 3 \mathrm{I}=4.02 \%+0.29 *$ Biweekly $+0.65 *$ Weekly $+1.18 *$ Twice+

1.90*Three+2.16*Supplement-0.10*Male+0.09*(Age/10)+0.45*Not USA

${ }_{3}$ We also considered a multivariable model which considered groups of non-US countries instead of a US vs. non-US variable. In this more complex model each region was compared to the US, with betas (95\% CIs) as follows: Asia $0.49 \%(0.26,0.73 \%) \dagger$, Europe $0.92 \%(0.40 \%, 1.45 \%) \dagger$, South America -0.01\% (-0.51\%, 0.51\%), Africa -0.17\% (-1.13\%, 0.78\%), Australia/NZ 0.32\% ($0.15 \%, 0.79 \%)$, Canada $0.95 \%(0.29 \%, 1.61 \%)^{*}$ and Unknown $0.05 \%(-0.68 \%, 0.76 \%)$. 
524 Table 5. Seventy-five percent 1 prediction intervals based on multivariable model from

525 study 22

\begin{tabular}{|l|l|l|}
\hline Fish consumption & Supplement & Not supplement \\
\hline Never & $6.61 \%(4.43,8.79)$ & $4.48 \%(2.30,6.65)$ \\
\hline Bi-weekly & $6.91 \%(4.74,9.09)$ & $4.78 \%(2.60,6.96)$ \\
\hline Weekly & $7.28 \%(5.10,9.46)$ & $5.14 \%(2.96,7.32)$ \\
\hline Twice weekly & $7.84 \%(5.66,10.02)$ & $5.71 \%(3.53,7.89)$ \\
\hline Three or more per week & $8.59 \%(6.41,10.77)$ & $6.46 \%(4.28,8.64)$ \\
\hline
\end{tabular}

526

527 1Seventy-five percent of people were within the range shown. For example, seventy-five percent

528 of people eating fish three or more times per week and taking an omega-3 supplement had O3I

529 values between 6.41 and $10.77 \%$.

530 2Based on model predicting O3I by supplement, fish consumption and age only. Predictions are 531 made for someone 50 years old.

532 


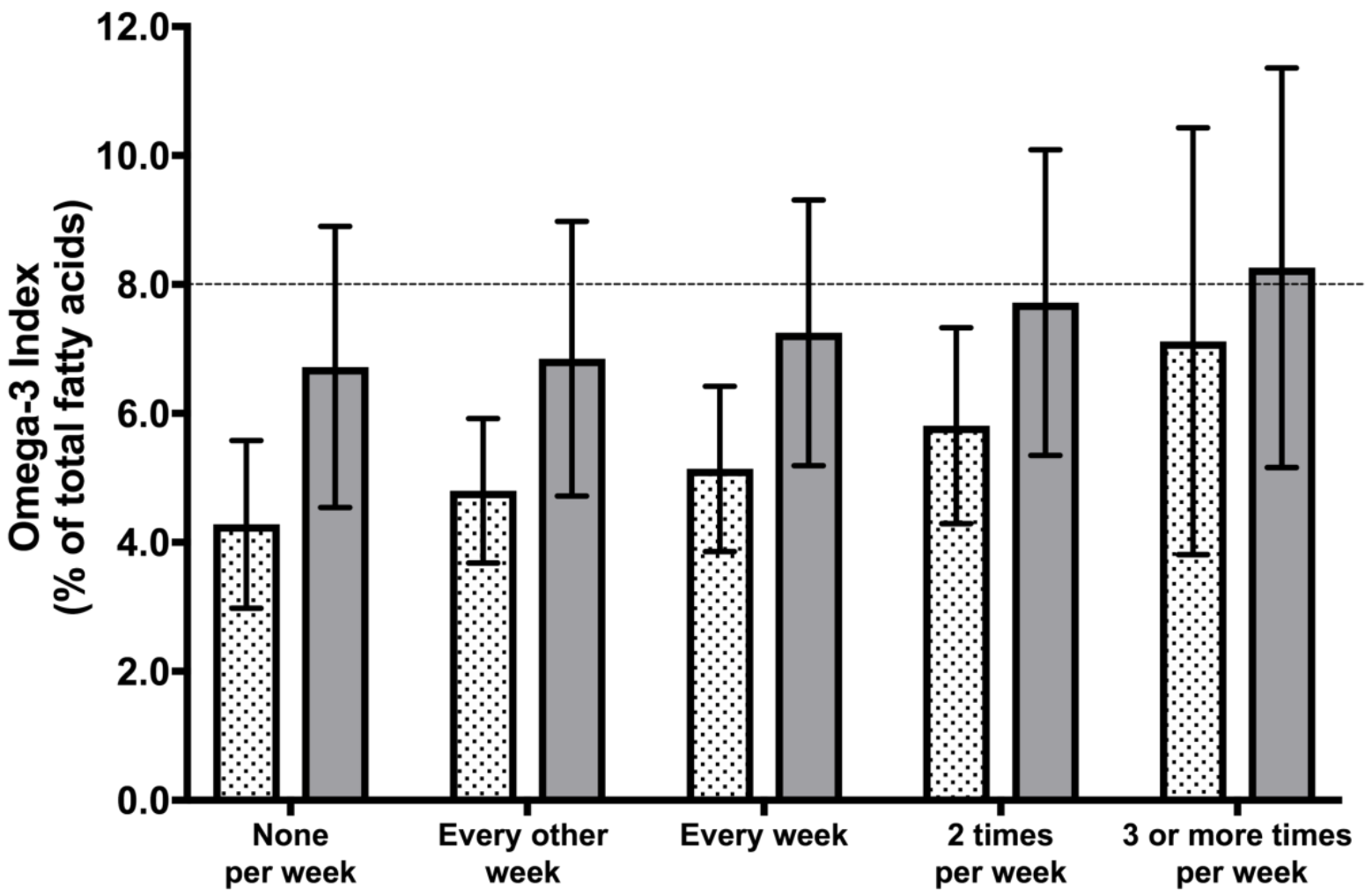

534 Figure 1. Omega-3 Index by fish intake and supplementation groups from Study 2 (mean \pm SD). 